\title{
Drug-Resistant Tuberculosis - Diagnosis, Treatment, Management and Control: The Experience in Thailand
}

\author{
Attapon Cheepsattayakorn \\ Additional information is available at the end of the chapter \\ http://dx.doi.org/10.5772/54852
}

\section{Introduction}

Multidrug-resistant tuberculosis (MDR-TB) is defined as TB bacilli revealing resistance to at least isoniazid and rifampicin whereas extensively drug-resistant tuberculosis (XDR-TB) is TB bacilli that develops resistance to at least isoniazid and rifampicin as well as to any quinolone drug and at least one of the second-line anti-TB injectable drug : kanamycin, amikacin, or capreomycin. Report from the World Health Organization (WHO)/International Union Against Tuberculosis and Lung Disease (IUATLD) Global Project on Drug Resistance Surveillance revealed that the prevalence of the primary multidrug-resistant tuberculosis during 1996-1999 ranged between 0-14.1 \% [1]. In 1994, the first WHO-IUATLD global anti-TB drug resistance surveillance was carried out in 35 countries and subsequently, the second, third and fourth surveillances occurred in 1996-1999, 1999-2002 and 2002-2007, respectively. The emergence of clinically significant MDR-TB was in the early 1990s. Reports of Primary DrugResistance Surveillance in Thailand during 1997-1998, 2001-2002, and 2005-2006 were $2.02 \%$ [2], $0.93 \%$ [3], and $1.65 \%$ [4], respectively while the secondary drug-resistance in 2005-2006 revealed $34.54 \%$ [4]. However, number of patients with MDR-TB demonstrated in 2008 Annual Report of the Bureau of Tuberculosis, Thailand were only 294 while the WHO' s estimated number of patients were 2,774 [5]. The prevalence of primary plus secondary MDR-TB among prisoners in Thailand in 2002-2003 was $5.3 \%$ while the prevalence of primary MDR-TB was $5.9 \%$ [6]. Report from the $10^{\text {th }}$ Zonal Tuberculosis and Chest Disease Centre, Chiang Mai, Thailand, $10^{\text {th }}$ office of Disease prevention and Control, Department of Disease Control, Ministry of Public Health, Thailand in 2011 revealed only 88 patients who registered with MDR-TB at hospitals in northern Thailand [7] while only $16.3 \%, 18.6 \%$ and $18.6 \%$ of 43 patients with laboratory confirmation were cure, completeness of treatment, and dead, respectively in 2009 report [8]. Findings from the Bureau of Tuberculosis of Thailand 's 2007-2009 Research 
Project on Anti-tuberculosis Drug Resistance Surveillance in Thailand (Situation of MultidrugResistant Tuberculosis in Thailand : Fiscal Year 2007-2009) that studied in 126 hospitals countrywide showed 877 patients with laboratory-confirmed MDR-TB and 64 patients with laboratory-confirmed XDR-TB while $21.5 \%$ were dead and $12.74 \%$ of these MDR/XDR-TB patients had human immunodeficiency virus (HIV) infection /acquired immunodeficiency syndrome (AIDS) compared to $21.57 \%$ of probable or presumptive MDR-TB patients coinfected with HIV/AIDS [9]. Only 18.2\% of the studied data sources came from TB registered book for MDR-TB patients and most of them came from the hospital medical registry [9]. A previous study by Scano F et al. revealed 52 of the 53 patients with XDR-TB died [10]. The median survival time from collection of specimen to death of these patients was 16 days [10]. The prevalence of XDR-TB among all MDR-TB patient was as the following : $10.3 \%$ in Germany and $14.3 \%$ in Italy (1993-2004) ; 1.5\% in Asia, $15.4 \%$ in Republic of Korea, $13.6 \%$ in Russia, $0.6 \%$ in Africa and Middle East, $6.5 \%$ in industrialized countries, and $6.6 \%$ overall worldwide (2000-2004); $12 \%$ in Hong Kong (2004); $10.9 \%$ in Iran (2006); $7.3 \%$ in India; and 4\% in France (2006) [11]. The WHO notified that Thailand possibly underreported of MDR/XDR-TB prevalence due to delaying of transportation of the specimens for anti-tuberculosis drug susceptibility testing to the specialized centres and processes of unstandardized data collecting of the country [9]. More than 400,000 new MDR-TB cases globally occur each year while approximately half of a million cases occurred in 2007 and accounted for more than $5 \%$ of the annually global cases of TB disease. The emerging of drug-resistant TB is a global health problem, although emphasis has been placed on several " hotspots " (higher than 3\% of its prevalence) worldwide because of lacking of good global data reported to the WHO. The emergence of MDR-TB and XDR-TB is a real health threat to achieve TB elimination.

\section{Epidemiology}

Development of anti-tuberculosis drug resistance can occur due to Mycobacterium tuberculosis genetic factor, previous anti-tuberculosis treatment-related factors and many other factors [11]. The mechanism of drug-resistance is shown in Figure 1. There is a constant rate of spontaneous mutation of 0.0033 mutations/deoxyribonucleic acid (DNA) replication that is unique for a diverse spectrum of prokaryotic organisms [12]. The average rates of spontaneous mutation for rifampicin, isoniazid, pyrazinamide, streptomycin, and ethambutol are $2.25 \mathrm{x}$ $10^{-10}, 2.56 \times 10^{-8}, 1 \times 10^{-3}, 2.95 \times 10^{-8}$, and $1.0 \times 10^{-7}$, respectively [13]. A previous study in India revealed $3.4 \%$ and $25 \%$ of primary and acquired MDR-TB, respectively [14] which were higher than the primary MDR-TB and acquired MDR-TB prevalence previously surveyed in Thailand $[2,3,4]$. Liu CH et al. reported their study in China which presented $19.4 \%$ of MDR-TB, $1.3 \%$ of XDR-TB, $19.8 \%$ of poly-resistant TB, and $47.1 \%$ of any anti-tuberculosis drug resistance [15]. A surveillance data in 2007 from the WHO demonstrated $4.8 \%$ of MDR-TB cases among new TB cases worldwide [16] compared to the study from MDR-TB surveillance in Thailand between 2007-2009 revealed the MDR-TB prevalence of 0-0.21\% (average $0.08 \%$ ) which was highest in the central part of Thailand [9]. The prevalence among patients with previously TB treatment from the same surveillance project was between $1.58-58.72 \%$ (average $8.49 \%$, higher 
than the percentage of hotspot of MDR-TB prevalence set by the $\mathrm{WHO}(3 \%$, an indicator for implementation of DOTS (Directly Observed Treatment, Short Course)-Plus programmes) which was also found highest in the central part of Thailand while the northern part of Thailand was the second [9]. This Thailand's serious MDR-TB situation needs urgently management such as implementation of DOTS-Plus programmes. In 2006, Gandhi NR et al. firstly reported of XDR-TB co-infected with HIV/AIDS which had been studied in Kwazulu Natal, South Africa (KZN) [11]. XDR-TB epidemic in South Africa appears to be the primary mechanism through the acquisition of $63-75 \%$ of XDR-TB cases [11] whereas the strain of Mycobacterium tuberculosis infected among a large number of XDR-TB cases in KZN was F15/LAM4/KZN [11]. Generally, individual who is infected with Mycobacterium tuberculosis has approximately $5-10 \%$ lifetime risk of developing TB disease, but in an individual with HIV-infection/AIDS the risk is $5-15 \%$ a year [11]. This can contribute HIV-infection/AIDS to facilitate the control of outbreaks of MDR-TB and XDR-TB, although it has contributed to outbreaks of drug-resistant TB [17]. The patients with MDR-TB and HIV/AIDS co-infection will have exceedingly high mortality [16]. Gandhi NR et al. recently reported their study on risk factors for mortality among MDR/XDR-TB patients with HIV/AIDS co-infection which revealed that $80 \%$ of XDRTB patients died whereas $63 \%$ of MDR-TB patients were dead following the diagnosis [18]. The CD4-T cell count less than 50 cells $/ \mathrm{mm}^{3}$ was the strongest independent factor for mortality among both patient groups [18]. History of TB treatment is the most significant predictor of development of MDR-TB [19]. High prevalence of HIV/AIDS co-infection and inadequate resources for case detection and management have contributed to the emergence of untreatable XDR-TB [20]. Unfortunately, the presence of XDR-TB in non-HIV-infected patients with MDR-TB is independent poor prognostic factors [11]. Prevalence of XDR-TB is globally accounted for approximately 5.4\% of MDR-TB prevalence [21]. Drug-resistant TB and drugresistant gram-negative bacterial infection and disease are associated with the most serious health problems in developing countries [22]. Estimated 81,000 patients with MDR-TB (18.4\% of the estimated MDR-TB patients worldwide in 2011) live in the 53 countries of the WHO European Region [23]. This European MDR-TB problem contributed to launching of a new WHO Regional Office for Europe Action Plan to fight MDR-TB to contain the spread of drugresistant TB in the region by the end of 2015 [23]. The new action plan set the targets to be achieved by the end of 2015 , are : 1 ) decreasing $20 \%$ of the proportion of MDR-TB cases among previously treated patients 2) diagnosis of at least $85 \%$ of the estimated MDR-TB cases and 3) treating successfully at least $75 \%$ of notified MDR-TB cases [23]. If this plan is fully implemented and expected, by 2015, to successfully treat 127,000 MDR-TB cases, and to prevent the emergence of 250,000 new MDR-TB cases and 13,000 new XDR-TB cases [23]. This would interrupt the transmission of MDR-TB and save 120,000 lives in this region [23].

\section{Systematic management of MDR/XDR-TB}

Diagnostic and treatment consultation networks for MDR/XDR-TB which set by the $10^{\text {th }}$ Zonal Tuberculosis and Chest Disease Centre, Chiang Mai, 10 $0^{\text {th }}$ Office of Disease Prevention and Control, Department of Disease Control, Ministry of Public Health, Thailand beyond the year 


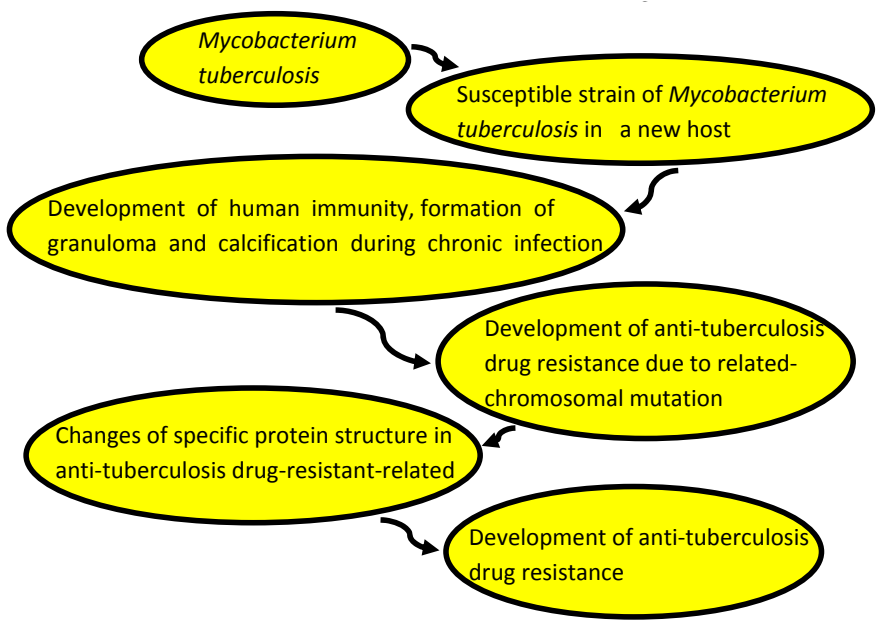

Figure 1. Mechanism of development of anti-tuberculosis drug resistance

2000 have been firstly initiated at tertiary care hospitals in northern Thailand. Data collecting on MDR/XDR-TB control has been tracked through the special project paper-based recording and reporting systems set by the $10^{\text {th }}$ Zonal Tuberculosis and Chest Disease Centre which separated from the routine DOTS recording and reporting systems. Currently, a new computer programme has been developed by staff of this centre to efficiently record and report the MDR/ XDR-TB data in the area of northern Thailand and attempt to gradually extend the use of this computer programme throughout the country.

\section{Presumptive diagnosis of MDR-TB}

The Thailand' s 2012 National Tuberculosis Management Guidelines [24] set the criteria for probable or suspected MDR-TB, are :

1. patients with history of TB retreatment, especially within 6 months after completeness of treatment or cure,

2. patients with interruption of 6 month-short course chemotherapy (2HRZE/4HR, H=isoniazid, $\mathrm{R}=$ rifampicin, $\mathrm{Z}=$ pyrazinamide, $\mathrm{E}=$ ethambutol) and having continuously positivesputum smear examinations after treatment interruption,

3. patients with history of multiple TB treatments, irregular anti-TB drug taking, and persistently positive-sputum smear examinations,

4. patients having positive-sputum smear examinations at the end of second and fifth months of the 6-month short course chemotherapy (2HRZE/4HR), 
5. patients with evidence of HIV co-infection/AIDS before the 6-month short course chemotherapy starting,

6. general TB patients with history of MDR-TB patient exposure, including health or medical personnel with TB disease who have history of MDR-TB patient exposure, and

7. other high-risk patients of MDR-TB, such as general TB patient with huge lung cavity, diabetic patients, prisoners with TB disease, and TB patients who live in the crossborderline areas.

\section{Laboratory investigations for MDR-TB}

Several plans have been announced for the WHO Stop TB Department to collaborate with the Foundation for Innovative New Diagnostics (FIND) to initiate and introduce rapid-culture technology and new rapid drug-resistant tests in the southern African countries and the world including the international standards for the second-line drug-susceptibility testing [11]. The Thailand' s 2012 National Tuberculosis Management Guidelines [24] set the laboratory investigations for MDR-TB which are direct acid-fast bacilli (AFB)-sputum smear examinations with sputum culture and drug susceptibility testing (DST). For reduction of the diagnosis time for unrecognized drug-resistant $\mathrm{TB}$, new rapid diagnostic technologies for drug resistance from sputum smear or positive culture for smear-negative and extra-pulmonary TB must be prioritized [10]. All TB control programmes in moderate- and high-MDR TB prevalent settings should consider the promotion of culture and DST including implementation of use of algorithms for the diagnosis of pulmonary and extra-pulmonary TB [10]. Rapid DST is preferred to the conventional DST due to 1-2 days of resulting. Recently, the $2011 \mathrm{WHO}$ Guidelines recommends "Xpert MTB/RIF ${ }^{\circledR}$ " and line-probe assay which are new molecular diagnostic technologies and can detect drug resistance to both isoniazid and rifampicin or only rifampicin [24], but their disadvantage is inability to detect the resistance to every drugs used in MDR-TB treatment for detecting probable XDR-TB, required expertise and expensive technologies/equipments which limit their wider uses [25]. The conventional DST takes 1-3 months of the results that take markedly longer than the new molecular methods and is laborintensive [24]. Other alternative phenotypic methods based on the Mycobacterium tuberculosis metabolism such as $\mathrm{CO} 2$ (carbon dioxide) production, oxygen uptake, ATP (adenosine triphosphate) bioluminescence, etc. have been experimented and demonstrated promising in overcoming this obstacle of longer time resulting [25]. These methods also have impressive sensitivity and specificity compared to the conventional DST [25]. Currently, molecular line probe assays and automated liquid culture systems are recommended by the WHO as the gold standard for the first-line and second-line DSTs. Liquid culture DST has been demonstrated to have relatively good reliability and reproducibility for aminoglycosides, fluoroquinolones, and polypeptides for detecting XDR-TB [26]. However, liquid culture DST for other secondline drugs such as para-aminosalisylic acid, linezolid, clarithromycin, amoxicillin-clavulanate, cycloserine, clofazimine, terizidone, ethionamide, and prothionamide is not recommended by the $\mathrm{WHO}$ [26]. A recent study reported the use of spoligotyping and sequence 6110 restriction 
fragment length polymorphism insertion analysis of genomic DNA which demonstrated that MDR-TB cases (74.0\%) were more likely to be identified in clusters than anti-TB drug susceptible cases (33.6\%) [27]. Xpert MTB/RIF assay recently has been introduced which meets the requirements of effective diagnosis of pulmonary TB as the following : allowing detection of both the Mycobacterium tuberculosis complex and resistance to the principal anti-TB drugs, especially rifampicin (RIF or R), availability on a global scale with standardized-easy use and robust diagnostic tools recently has been introduced [28]. This assay is a nucleic acid amplification test for detection of rifampicin resistance-associated mutations of the rpoB gene and Mycobacterium tuberculosis complex DNA in sputum [28]. It can be designed for use with other systems to automate and integrate sample processing, nucleic acid amplification, and detection of target sequences using reverse transcriptase polymerase chain reaction (PCR) and real-time PCR [28]. Between 2007-2009, the WHO has approved several drug-resistant TB diagnostic tests such as liquid culture $($ MGIT®, an automated liquid culture, developed by BD Diagnostic Systems, 2007) which has been used at the $10^{\text {th }}$ Zonal Tuberculosis and Chest Disease Centre, Chiang Mai, Thailand, line-probe assays (INNO-Lipa ${ }^{\circledR}$, line-probe assay that requires culture, developed by Innogenetics, 2008), noncommercial culture and drug susceptibility testing (Microscopic Observation Drug Susceptibility (MODS), developed by Academic Laboratories, 2009; Nitrate reductase assay, developed by Academic Laboratories, 2009; and Colorimetric drug susceptibility testing, developed by Academic Laboratories, 2009) [29]. GeneXpert MTB/ RIF®, a new automated nucleic acid amplification technique which was developed by Cepheid, The Foundation for Innovative New Diagnostics (FIND) and University of Medicine and Dentistry of New Jersey (UMDNJ) was reviewed by the WHO in 2011 [29] and currently has been recommended to measure the Mycobacterium tuberculosis DNA and the rifampicinresistance sequence worldwide. This new technique has been set in Thailand at least 6 sets including the one set at the $10^{\text {th }}$ Zonal Tuberculosis and Chest Disease Centre, Chiang Mai, Thailand in collaboration with the Unites States Centres for Disease Control and Prevention (US-CDC) for reference laboratories in Thailand. GeneXpert test's sensitivity is moderate at $67.2 \%$ in AFB-smear negative cases at one-time smear staining of the specimens, and increases to $80 \%$ when is performed three times [28]. This test provides the results within two hours and requires minimal training of the laboratory workers [29]. The limitations of the test are requirement of a consistent source of electricity that will limit its use outside of the settings where a regular electric power supply can be guaranteed, its expensive cost of the instrument, and cost per test cartridge [29]. Mishra B et al. used the automated BACTEC 460 TB system in study the emergence of drug-resistant TB at an urban tertiary care hospital in South India which revealed that $37.2 \%$ were MDR-TB isolates whereas $42 \%$ of the pulmonary Mycobacterium tuberculosis isolates and $20.4 \%$ of extra-pulmonary isolates were MDR [30]. Phenotypic and genotypic detections of anti-TB drug resistance are described as the following [31- 49]:

\section{Phenotypic detection}

\subsection{Slide DST}

This method has less equiptment, suitable for decentralization, 93\% rifampicin susceptibility at $88 \%$ predictive value of resistance.

\subsection{Mycobacteria Growth Indicator Tube (MGIT) Systems}


This test has sensitivities of $100 \%$ for rifampicin, isoniazid, ethambutol and streptomycin, specificity of $100 \%$ for rifampicin, $97.7 \%$ for isoniazid, $98.0 \%$ for ethambutol and $89.8 \% \%$ for streptomycin.

\subsection{Microscopic observation broth-drug susceptibility assay (MODS)}

This method has sensitivity of $72.7 \%$ and specificity of $99.7 \%$ for rifampicin, $72.6 \%$ and $97.9 \%$ for isoniazid, and $77.8 \%$ and $99.7 \%$ for MDR-TB.

\subsection{Mycobacteriophage-based method}

\subsubsection{Commercial FASTPlaque assay (FASTPlaque TB test and FastPlaque TB-} RIF $^{\mathrm{TM}}$ (rifampicin DST) (Biotech Labs Ltd, Ipswich, UK))

This test has sensitivity and specificity of $31.2 \%$ and $94.9 \%$, respectively in all anti-TB drugsusceptible and resistant TB patients, sensitivity and specificity of $33.3 \%$ and $93.9 \%$, respectively in all anti-TB drug-susceptible and resistant TB patients with HIV-infection/AIDS.

\subsubsection{Fluoromycobacteriophage assay (Figures 2,3)}

This method has 94\% sensitivities for rifampicin and isoniazid and 98\% sensitivity for streptomycin and specificities of $97 \%$ for isoniazid, $95 \%$ for rifampicin, and $98 \%$ for streptomycin (resazurin microplate technique), sensitivity of $94 \%$ for all three anti-TB drugs (rifampicin, isoniazid, and streptomycin) (EGFP-phage technique) and specificities of $93 \%$ for rifampicin, 90\% for isoniazid and 95\% for streptomycin (EGFP-phage technique).

\subsubsection{Luciferase reporter phage assay}

This test has 100\% sensitivity and $89-100 \%$ specificity for culture isolates.

\subsection{Nitrate reductase assay}

This method has sensitivity and specificity of $100 \%$ and $100 \%$ for rifampicin, $93 \%$ and $100 \%$ for isoniazid, $76 \%$ and $100 \%$ for streptomycin, and 55\% and $99 \%$ for ethambutol, respectively.

\subsection{Microcolony method (Thin-layer agar (TLA) method)}

This test has sensitivities and specificities of $100 \%$ for both rifampicin and ofloxacin, and sensitivity of $100 \%$ and specificity of $98.7 \%$ for kanamycin, $100 \%$ overall accuracy for rifampicin and isoniazid resistance.

\subsection{Colorimetric redox indicator methods}

This method has sensitivities of $100 \%$ for rifampicin, ofloxacin, kanamycin and capreomycin and $99.1 \%$ for isoniazid, specificity of $100 \%$ for rifampicin, isoniazid, ofloxacin and kanamycin, and $97.9 \%$ for capreomycin, overall accuracy of $98.4 \%$ for rifampicin, $96.6 \%$ for isoniazid, $96.7 \%$ for ofloxacin, $98.3 \%$ for kanamycin and $90 \%$ for capreomycin.

\section{Genotypic detection (Nucleic Acid Amplification)}

2.1 Line-probe assay (LPA)

\subsubsection{INNO-LiPA Rif.TB® assay (Innogenetics, Ghent, Belgium)}


This test has higher than $95 \%$ sensitivity and $100 \%$ specificity, sensitivity of $82.2 \%$ and specificity of $66.7 \%$ for MDR-TB detection.

\subsubsection{GenoType ${ }^{\circledR}$ MTBDRplus kit (Hain Lifescience, Nehren, Germany)}

This method has nearly 91\% sensitivity for MDR-TB, possibly detects rifampicin and isoniazid resistance and confirms TB infection simultaneously.

\subsubsection{Genotype MTBDRsl assay}

This test has ethambutol, $89 \%$ sensitivity for ofloxacin, $87 \%$ sensitivity for capreomycin, $75 \%$ sensitivity for amikacin.

\subsection{Real-time PCR}

This technique has sensitivity of $89 \%$ and specificity of $99 \%$ (molecular beacons), overall sensitivity of $98-100 \%$ with $72 \%$ sensitivity in smear-negative specimens and specificity of $100 \%$ for Xpert MTB/RIF assay (Cepheid Xpert MTB/RIF®, Sunyvale, CA).

\subsection{PCR sequencing}

This technique has sensitivities of $96.7 \%$ for rifampicin-resistant isolates ( $r p o B$ gene), $64 \%$ for isoniazid-resistant isolates ( $k a t G$ gene) and $70 \%$ of ofloxacin-resistant isolates (gyr $A$ gene) (PCR pyrosequencing).

\subsection{DNA Microarrays (DNA biochip)}

This technique has specificity of $97 \%$ and $95 \%$ for rifampicin, $91 \%$ and $60 \%$ for isoniazid, $96 \%$ and $67 \%$ for kanamycin, $93 \%$ and $73 \%$ for streptomycin, and $98 \%$ and $89 \%$ for ethambutol, respectively, simultaneous detection of multiple genetic sequences (oligonucleotide microarray).

Although genotypic methods have potentially fastest results, the massive cross-contamination is still the main risk. To prevent its, strict internal controls, special technique, and separation of working areas are required. The reproducibility of the results of the Xpert MTB/RIF assay under actual field conditions, the strength of the laboratories, and the manner and extent of its introduction are the impact of this new assay. The sources of error for genotypic methods are incomplete coverage of rifampicin-resistance gene-core region and mixtures (multiple mutations, wild-type strain/emerging mutant) and silent mutations. These sources of error may contribute to $1 \%$ false-resistant and $5 \%$ false-susceptible results [31].

Schematic representation of phAE87 : hsp60-EGFP construction. Shuttle phasmid phAE87 is a conditionally replicating derivative of phage TM4 in which the cosmid moiety is flanked by Pac I restriction sites. A plasmid derivative of $p Y U B 854$ containing the EGFP gene (pMP14) was used to replace the cosmid in $p h A E 87$ followed by lambda packaging and recovery in E. coli. 


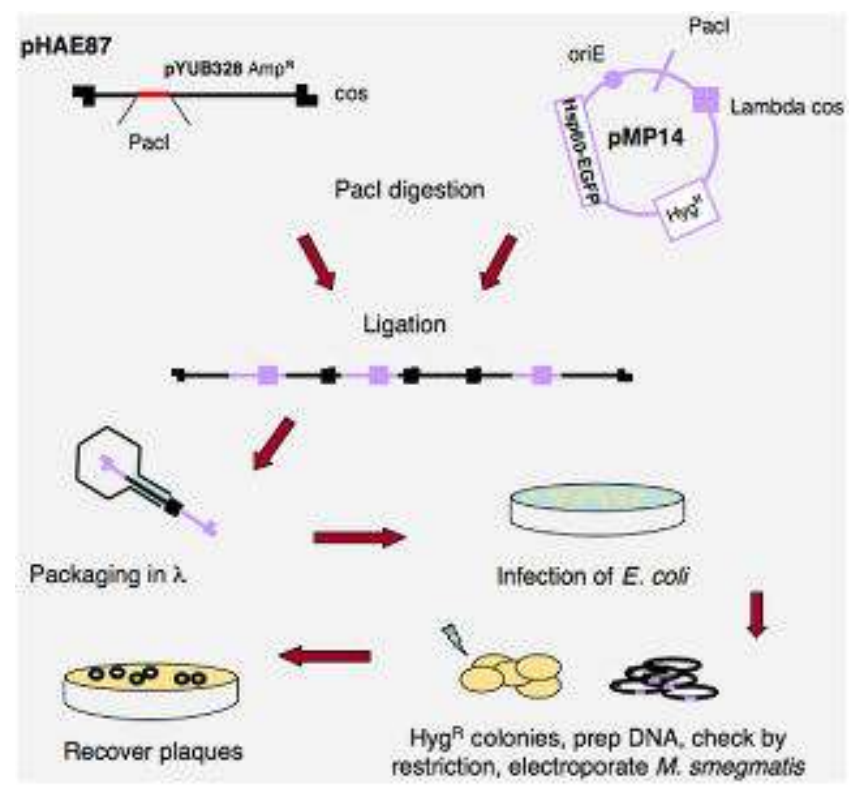

Source : Piuri M, Jacobs WR Jr, Hatfull GF. Fluoromycobacteriophages for rapid, specific, and sensitive antibiotic susceptibility testing of Mycobacterium tuberculosis. PLoS ONE 2009; 4(3) : e4870. doi: 10.1371/journal.pone.0004870

Figure 2. Fluoromycobacteriophages construction
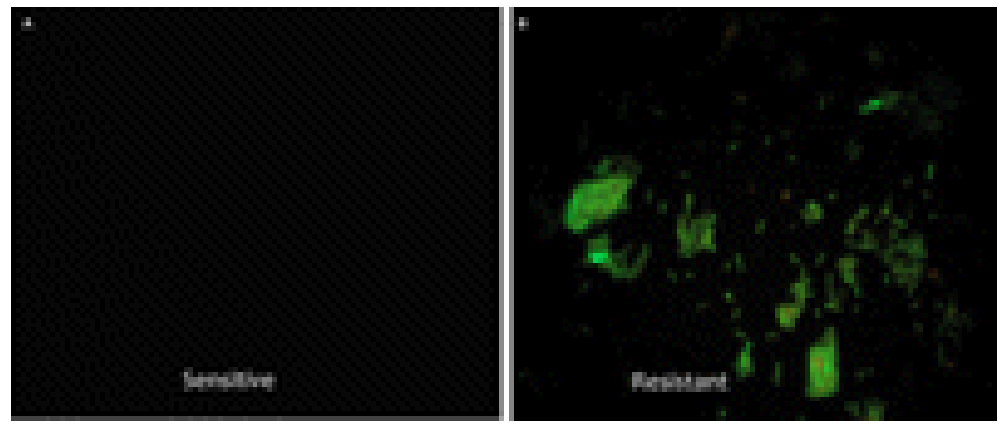

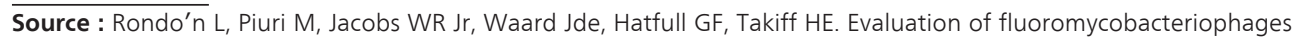
for detecting drug resistance in Mycobacterium tuberculosis. J Clin Microbiol 2011; 49 (5) : 1838-1842.

Figure 3. A, B). Two strains of Mycobacterium tuberculosis were incubated separately in 7H9-OAD with $2 \mu \mathrm{g}$ of rifampicin/ml for 24 hours, infected with the EGFP-phage, killed with paraformaldehyde, and then fixed on microscope slides. The images, obtained with a fluorescence microscope, show a susceptible-to-rifampicin strain (A) and a resistant-to-rifampicin strain (B). 


\section{Radiological features in patients with MDR/XDR-TB}

A recent study in South Korea showed 100\% of lung nodules, $60 \%$ of lung consolidation, and $47 \%$ of lung cavities that were mainly located in the upper and middle lung zones in XDR-TB patients whereas less frequent lung nodules and ground-glass opacity lesions were found in XDR-TB patients compared to the patients with anti-TB drug-susceptible pulmonary TB [50]. More frequent multiple lung cavities, lung nodules, and bronchial dilatation were found in both MDR-TB and XDR-TB patients compared to the patients with anti-TB drug-susceptible pulmonary TB [50]. There was no different radiological findings between MDR-TB and XDR-TB patients [50]. Another recent study in South Korea revealed that micronodules and tree-in-bud appearance were found in $100 \%$ of the pulmonary XDR-TB patients whereas lung consolidations, lung cavities, bronchiectasis, lobar consolidations were found in $85 \%, 85 \%, 80 \%$, and $70 \%$, respectively [51]. This study showed a significantly larger extent of tree-in-bud appearance and lung consolidations compared to the MDR-TB patients [51]. In childhood patients, chest radiological features at the time of diagnosis demonstrates lobar opacification, intrathoracic lymphadenopathy, particular hilar lymph nodes, and airway narrowing [52]. Chest radiological features of three patients with MDR-TB who attended the $10^{\text {th }}$ Zonal Tuberculosis and Chest Disease Centre, Chiang Mai, Thailand are shown in the Figure $4(\mathrm{~A}, \mathrm{~B}, \mathrm{C})$ which demonstrated a single cavity at the upper lung zone in two patients and no lung cavity in another one. These three patients possibly attended the $10^{\text {th }}$ Zonal Tuberculosis and Chest Disease Centre, Chiang Mai, Thailand at the earlier stages compared to the above study results.
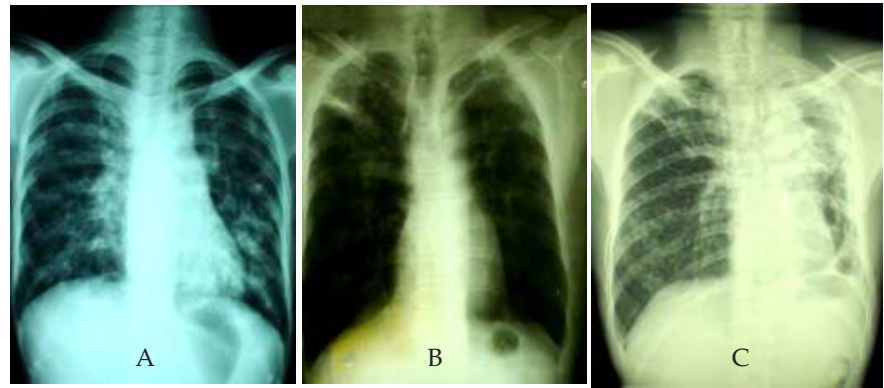

Figure 4. First-attendance chest radiological features of the three patients $(A, B, C)$ with MDR-TB at the $10^{\text {th }}$ Zonal Tuberculosis and Chest Disease Centre, Chiang Mai, Thailand A : Showing bilaterally diffuse lung infiltration with a cavity in the left upper lung zone, B : Showing fibrotic infiltration with surrounding new infiltration at the right upper lung zone with bilaterally diffuse emphysematous lung changes, C : Showing bilaterally diffuse infiltration with a cavity in the right upper lung zone with left pleural effusion. 


\section{Regimens used in treating patients with drug-resistant tuberculosis}

A recent study on MDR-TB treatment revealed that use of later generation quinolones (moxifloxacin, gatifloxacin, sparfloxacin, levofloxacin), ofloxacin or ethionamide/prothionamide, use of four or more likely effective drugs in the initial intensive phase, and three or more likely effective drugs in the continuation phase was associated with the treatment success compared to the treatment failure or relapse [53]. The duration of initial phase up to 7.1-8.5 months and the total duration of treatment up to 18.6-21.5 months increased the chances of treatment success [53]. In 2011, the WHO recommended the regimens containing a fluoroquinolone, pyrazinamide, ethionamide (or prothionamide), para-aminosalicylic acid (or cycloserine), and a second-line injectable drug with more than 20 months of treatment duration [54]. Five MDR-TB control projects with used DST results and previous treatment history were conducted among 1,047 MDR-TB patients in 5 resource-limited settings with well-established DOTS programmes (Manila, Estonia, Latvia, Lima, and Tomsk) in 1999 for Lima and Manila, 2000 for Tomsk and Latvia, and 2001 for Estonia [55]. At least 4 drug (ethambutol, pyrazinamide, cycloserine, clofazimine, para-aminosalicylic acid, ethionamide, or prothionamide, augmentin, clarithromycin or thiacetazone) including an injectable drug (kanamycin, amikacin, capreomycin, or streptomycin) and a fluoroquinolone (ofloxacin, ciprofloxacin, or levofloxacin) were administered for the duration of treatment (18-24 months) except for the injectable drug, which was administered for a specified interval after the patient' s specimens were culture-negative [55]. Monthly sputum-AFB smear and culture were monitored [55]. Every 6-months (Manila and Lima) and 3-months chest radiographs (Tomsk, Latvia, and Estonia) were performed [55]. The treatment outcomes among new and previously treated MDR-TB patients revealed $74.8 \%$ and $68.3 \%$ cured patients, $2.5 \%$ and $0.3 \%$ completed treatment patients, $4.2 \%$ and $7.0 \%$ failed treatment patients, $3.4 \%$ and $14.2 \%$ dead patients, and $77.3 \%$ and $66.6 \%$ treatment success rates (cure rate + completed treatment rate), respectively [55]. The results showed worsen outcomes among previously treated patients. Report from the $10^{\text {th }}$ Zonal Tuberculosis and Chest Disease Centre, Chiang Mai, Thailand, in 2011 which had been collected from the data of laboratory-confirmed 254 MDR-TB patients $(72.8 \%$ of all probable MDR-TB cases) among 349 totally suspected-MDR-TB cases with $15.8 \%$ of HIV coinfection in northern Thailand between 2005-2010 gradually increased from $62.2 \%$ of probable MDR-TB cases in 2005 to $78.3 \%$ in 2010 and revealed 3.2\% treatment-denial patients, $75.2 \%$ treatment-registered patients, $30.2 \%$ died before starting the second-line drug treatment regimens (pyrazinamide, ethambutol, ofloxacin, para-aminosalicylic acid administered for 18-24 months and one injectable drug (kanamycin, or amikacin) administered 5 days per week for the initial 6-month phase), 25.4\% default-treatment patients (continuous interruption of treatment more than 2 months), 54.8\% treatment success rate, and $22.2 \%$ unavailable-data patients [7]. Among 19 cases with pre-treatment death, 10 cases (52.63\%) demonstrated HIV co-infection. Extra-pulmonary cases accounted for $2.4 \%$ of the laboratory-confirmed cases which was lower than percentage of susceptible extra-pulmonary TB cases in the same area [56]. Four cases with laboratory-confirmed MDR-TB emerged as XDR-TB during treatment [7]. A previous study on outcomes of a daily supervised-MDR-TB treatment regimen which consisted of initial phase of 6-9 months with kanamycin, ofloxacin, cycloserine, ethionamide, 
ethabutol, and pyrazinamide demonstrated that in cases of persistent culture positive at fourth month, the initial phase was extended for additional 3 months. Then ofloxacin, cycloserine, ethionamide, and ethambutol were continued for 18 months [57]. The results of the study revealed that $82 \%$ of cases demonstrated time to culture conversion at the second month or before. The culture conversion rates at third month and sixth month were $84 \%$ and $87 \%$, respectively. The cure rate was $66 \%$. At 24 months, $79 \%$ of the patients remained culture negative for more than 18 months. Adverse drug reactions were reported among 58\% of cases and 2 failure cases emerged as XDR-TB during treatment [57]. A recent study on comparison between traditional hospital-based treatment-model of MDR-TB patient care and communitybased model in rural areas of South Africa revealed that median times to starting the treatment and sputum smear conversion were shorter for community-based model (84 days versus 106.5 days and 59 days versus 92 days, respectively) [58]. Lack of sputum culture conversion at month 9 was a predictor of pulmonary MDR-TB treatment failure with $84 \%$ of sensitivity and $94 \%$ of specificity [59]. A recent study by Dheda K et al. demonstrated that the number of XDRTB deaths was not significantly different compared between patients with and without HIV co-infection [60] whereas Well CD recently reported that lower cure rates and higher death rates were found in MDR-TB patients with HIV co-infection compared to the patients without HIV co-infection [61]. Survival of XDR-TB patients with HIV co-infection was associated with absence of biomarkers indicative of multiorgan dysfunction, less advanced stage of both diseases at time of diagnosis, and antiretrovirals provision [62]. Previous culture-proven MDRTB, number of drug used in a MDR-TB treatment regimen, and treatment with moxifloxacin were independent predictors of death [60] while some previous reports showed that treatment success rates were poor (30-50\%) in XDR-TB patients with HIV co-infection [63] and the MDR/ XDR-TB prevalence was substantially precipitated by the HIV epidemic [64, 65]. In children with MDR-TB, the treatment guidelines are the same principles, using the same drugs as in adult patients with strict and prolonged supervision by expert pediatricians [66]. HIV coinfection are particular challenges and requires early starting of antiretroviral therapy with careful monitoring for drug-adverse side-effects [66]. Children with close contact with MDRTB patients should be tested with tuberculin skin testing or interferon-gamma release assays, direct AFB-smear examinations, cultures, and DST and taking the chest radiological examinations [24]. Cases with close contact should be at least 2-year followed up [24]. If they are diagnosed MDR-TB, they must be treated with the empirical MDR-TB regimen [24]. Empirical MDR-TB treatment regimen is not recommended for MDR-TB chemoprophylaxis [24]. MDRTB chemoprophylaxis for children with at least 2 second-line drugs for 6-12 months and reflecting the susceptibility profile of the source case' $\mathrm{s}$ isolate with daily supervision should be considered [52,67]. The knowledge of mechanisms of the second-line drugs for children is necessary for ensuring the treatment adherence and long-term control of the disease.

A previous study on the second-line drug susceptibility among 40 MDR-TB strains in Turkey revealed mono-resistant to ethionamide $25 \%$, amikacin $10.0 \%$, kanamycin $2.5 \%$, ofloxacin $2.5 \%$, amikacin $0 \%$, and clofazimine $0 \%$, any resistant to ethionamide $37.5 \%$, capreomycin $25.0 \%$, kanamycin $5.0 \%$, ofloxacin $5.0 \%$, amikacin $5.0 \%$, and clofazimine $2.5 \%$, resistant to both ethionamide + capreomycin $5.0 \%$, both capreomycin + clofazimine $2.5 \%$, ofloxacin + ethionamide + capreomycin $2.5 \%$, amikacin + ethionamide + capreomycin $2.5 \%$, and kanamycin + 
amikacin + ethionamide + capreomycin 2.5\% [68]. Currently, the data of the second-line drug resistance are not available [9].

The Thailand' s 2012 National Tuberculosis Management Guidelines [24] recommends the guidelines for both pulmonary and extra-pulmonary MDR-TB treatment as the following flow diagram :

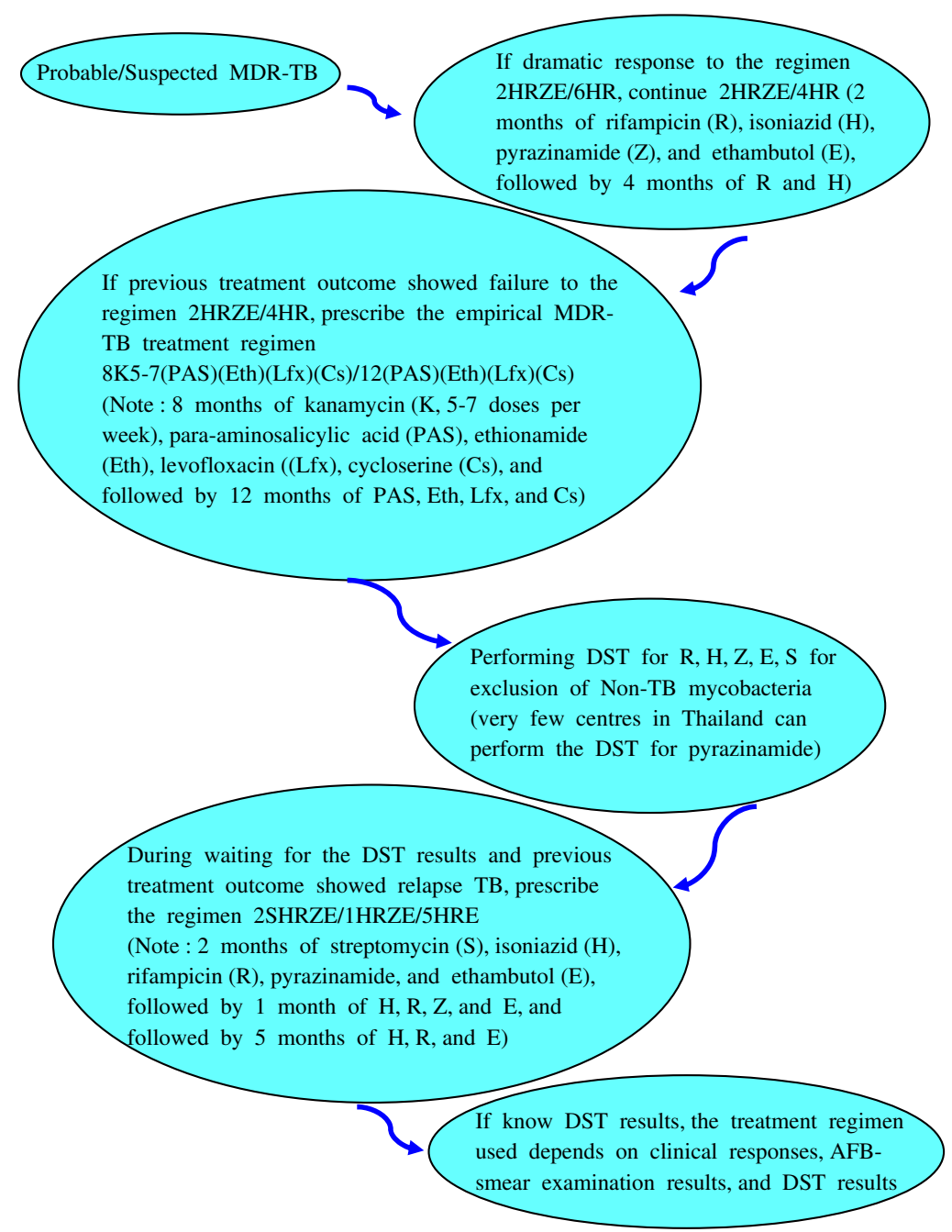

Figure 5. Flow Diagram of Management of Patient with Probable/Suspected MDR-TB

Currently, in Thailand, patients with persistent AFB-smear and/or culture positive after completeness of MDR-TB treatment will be prescribed isoniazid alone for lifelong while no 
standardized treatment is recommended yet. Types of patients with MDR-TB, patient monitoring during MDR-TB treatment and assessment of sputum conversion, and classification of treatment outcomes are shown in the Table 1, 2, and 3, respectively [24].

\begin{tabular}{|c|c|}
\hline Type & Description \\
\hline New case & $\begin{array}{l}\text { patient who does not take any anti-TB drugs before or take any anti-TB drugs less than } 1 \text { month } \\
\text { duration and the DST results show resistance to at least rifampicin and isoniazid }\end{array}$ \\
\hline Relapse case & $\begin{array}{l}\text { patient who cured of MDR-TB in the past and present or pre-treatment of relapse-MDR-TB DST } \\
\text { results reveal MDR-TB }\end{array}$ \\
\hline $\begin{array}{l}\text { After default MDR- } \\
\text { TB }\end{array}$ & $\begin{array}{l}\text { patient who continuously interrupts the TB treatment more than } 2 \text { months and the DST results } \\
\text { before treatment interruption demonstrates MDR-TB }\end{array}$ \\
\hline $\begin{array}{l}\text { After failure of the } \\
\text { first- TB treatment }\end{array}$ & $\begin{array}{l}\text { patient' s AFB examinations show positive results at the end of the fifth month and the DST } \\
\text { results at the end of the second or fifth month reveal MDR-TB }\end{array}$ \\
\hline $\begin{array}{l}\text { After failure of } \\
\text { retreatment }\end{array}$ & $\begin{array}{l}\text { patient who treated with the retreatment regimen ( } 2 \text { months of streptomycin, rifampicin, } \\
\text { isoniazid, pyrazinamide, and ethambutol, followed by } 1 \text { month of rifampicin, isoniazid, } \\
\text { pyrazinamide, and ethambutol, and then followed by } 5 \text { months of isoniazid, pyrazinamide, and } \\
\text { ethambutol for relapse cases) and the DST results at the end of third or fifth month demonstrate } \\
\text { MDR-TB }\end{array}$ \\
\hline Transfer in & $\begin{array}{l}\text { patient who is referred from one setting to the other setting for further diagnosis, treatment, } \\
\text { and management, transferred setting must report the outcome of treatment to the referred } \\
\text { setting (first setting) for discharge registration as "transfer in " }\end{array}$ \\
\hline Other & $\begin{array}{l}\text { other patients who cannot be registered as the above } 6 \text { patient-registration types including } \\
\text { patients who treated with empirical MDR-TB treatment regimen at the non-NTP settings (not } \\
\text { registered as the above } 6 \text { patient-registration types) }\end{array}$ \\
\hline
\end{tabular}

Table 1. Registration of the patients with MDR-TB [24]

\begin{tabular}{cr}
\hline Patient monitoring during MDR-TB treatment & Assessment of sputum conversion \\
\hline $\begin{array}{c}\text { Direct AFB-smear examinations and cultures will be } \\
\text { performed at month : } 0,1,2,3,4,5,6,7,8,10,12,14,\end{array}$ & $\begin{array}{r}\text { Results of } 2 \text { consecutively direct-AFB-smear negative and } \\
\text { culture negative with 30-day intervals will indicate sputum } \\
\text { conversion. Mostly, patients with sputum conversion will }\end{array}$ \\
$\begin{array}{c}\text { Chest radiological examinations will be performed at } \\
\text { month : } 0,3,6,12, \text { and } 18 .\end{array}$ & $\begin{array}{c}\text { converse the positive to the negative results within the first } 6- \\
\text { mont of treatment duration. }\end{array}$ \\
\hline
\end{tabular}

Table 2. Patient monitoring during MDR-TB treatment and Assessment of sputum conversion [24]

\section{Patient monitoring after completeness of the MDR-TB treatment [24]}

For assessment of the MDR-TB relapse rate, direct AFB-smear examinations and cultures are performed and assessed every 3 months in the first 6-month completeness of treatment and 
then every 6 months for 18 months. Chest radiological examinations are preformed when indicated.

\begin{tabular}{|c|c|}
\hline Cured & $\begin{array}{l}\text { patient who treated with MDR-TB treatment regimen following the Thailand's } 2012 \text { National } \\
\text { Tuberculosis Control Management (or NTP) Guidelines with consecutively 5- negative results of the } \\
30 \text { day-interval AFB smear examinations and cultures of the last } 12 \text { months of treatment duration }\end{array}$ \\
\hline $\begin{array}{l}\text { Treatment } \\
\text { completed }\end{array}$ & $\begin{array}{l}\text { patient with completeness of the MDR-TB treatment following the Thailand's } 2012 \text { NTP } \\
\text { Guidelines but no consecutively AFB-smear examination and culture results during the last 12- } \\
\text { month treatment duration }\end{array}$ \\
\hline Died & $\begin{array}{l}\text { patient with any cause of death during MDR-TB treatment following the Thailand's } 2012 \text { NTP } \\
\text { Guidelines }\end{array}$ \\
\hline Failed & $\begin{array}{l}\text { patient treated with MDR-TB regimen following the Thailand's } 2012 \text { NTP Guidelines with } 2 \\
\text { positive results of the } 5 \text { consecutive AFB-smear examinations and cultures during the last } 12 \\
\text { months of treatment duration or patient with } 1 \text { positive-culture result of the last } 3 \text { consecutive } \\
\text { cultures or patient with physician's decision to stop the MDR-TB treatment due to clinical } \\
\text { unresponsiveness or various adverse-drug reactions }\end{array}$ \\
\hline Default & $\begin{array}{c}\text { patient with continuous interruption of the MDR-TB treatment following the Thailand's } 2012 \text { NTP } \\
\text { Guidelines }\end{array}$ \\
\hline Transfer out & $\begin{array}{l}\text { partially MDR-TB treated patient with referring from (Transferring out) one setting to another } \\
\text { setting with unknown treatment outcomes will be registered as "Transfer out" }\end{array}$ \\
\hline
\end{tabular}

Table 3. Classification of treatment outcomes [24]

\section{MDR-TB management and treatment outcomes in Thailand between 2007-2009}

The prevalence of laboratory-confirmed MDR-TB was $0.08 \%$. Highest prevalence $(0.21 \%)$ was found in the central part of Thailand. MDR-TB was mostly diagnosed and treated at the secondary care settings or general hospitals (31.5\% and $31.14 \%$ ), $24.3 \%$ and $25.08 \%$ of cases were diagnosed at the tertiary care settings and only $6.9 \%$ and $6.7 \%$ of the patients were diagnosed at the university hospitals, respectively [9]. The majority of the patients $(63.82 \%)$ were registered as " after failure of the first-TB treatment " [9]. In Thailand, numbers of the secondary care settings or general hospitals are more than that of the tertiary care hospitals, this may reflex the above figures. Only $33.5 \%$ of cases were referred to the well-facilitated setting for directly observed treatment (DOT) [9]. Only 60.6\% of MDR-TB cases were prescribed 4 oral second-line drugs and an injectable aminoglycoside drug [9] which recommended by the Thailand' s 2012 National Tuberculosis Management Guidelines while the rests were prescribed various treatment regimens [24]. Only $57.5 \%$ of cases had completed treatment adherence [9]. Low DOT implementation can contribute to high default rates, high treatment failure rates, high death rates, and low cured rates. There was $24.2 \%$ of patients with com- 
pleteness of treatment, $29.1 \%$ cured, $20.5 \%$ default, $2.2 \%$ treatment failure, and $21.5 \%$ died [9]. Treatment failure and treatment default rates were higher among new case compared to the patients with previous TB treatment whereas higher death rates were found among the patients with previous TB treatment. This could be due to inadequately strict- and intensivehealth education provision to the new cases and more severe disease at the time of diagnosis among the patients with previous TB treatment. Only $27.5 \%$ of cases with completed treatment were followed up more than 2 months [9].

A recent study in South Korea revealed that the treatment regimen was individualized based on the history of anti-TB drugs taken by the patient and the most DST result [69]. Three to seven anti-TB drugs were self-administered except injectable drugs during hospitalization [69]. Injectable drugs were prescribed for 6-7 months [69]. The total treatment duration was at least 18 months after sputum culture conversion [69]. If the medical treatment was expected to fail or had failed in patients with localized lung cavities, or bilateral lesions and anticipated adequate postoperative lung function, surgical resection was considered [69]. The treatment outcomes showed that $37.1 \%$ of patients had treatment success, and $4.5 \%$ of them died of all causes during the 3-4 years after treatment initiation [69]. The independent predictors of allcause mortality were age, history of MDR-TB treatment, XDR-TB, and prothionamide resistance [69]. Currently, there is no DOTS programme implementation in South Korea [69] while Thailand has been implemented several years ago, but the treatment outcomes were better than that of Thailand $[9,69]$. These different results of both projects should be intensively investigated and explained. Kliiman $\mathrm{K}$ et al. recently demonstrated that predictors of poor treatment outcomes in MDR-TB were previous TB treatment, ofloxacin resistance, positiveAFB smear, and HIV-infection/AIDS [70].

The criteria for capacity of establishment of the specialized MDR-TB centre that recommended by the Thailand' s 2012 NTP guidelines [24] are as the following : 1.authorized persons' recognition of the MDR-TB threats 2.good laboratory networks and good patient-referral system 3.good DOT system, and 4.consistently continuous care for MDR-TB patients.

\section{XDR-TB treatment}

A previous study in Peru by Mitnick CD et al. demonstrated that 48 (7.4\%) of 651 tested-isolate patients had XDR-TB [71]. The results showed various individualized regimens prescribed for 47 patients with XDR-TB as the following : 1) $14.9 \%$ of the patients included ethambutol, 2) $34.0 \%$ included pyrazinamide, 3) 38.3\% included streptomycin, 4) 19.1\% included amikacin, 5) $53.2 \%$ included capreomycin, 6) $17.0 \%$ included kanamycin, 7) 21.3\% included ciprofloxacin, 8) $12.8 \%$ included ofloxacin, 9) $42.6 \%$ included levofloxacin, 10) $2.1 \%$ included spafloxacin, 11) 72.3\% included moxifloxacin, 12) 100\% included cycloserine, 13) 66.0\% included ethionamide, 14) $95.7 \%$ included para-aminosalicylic acid, 15) $100 \%$ included amoxicillin-clavulanate, 16) $44.7 \%$ included clarithromycin, 17) $97.9 \%$ included clofazimine, 18) $17.0 \%$ included rifabutin, 19) number of drugs in regimen (number without documented resistance or prior exposure for more than 1 month) : 19.1) $5.3+/-1.3$ agents among all available agents, 19.2) $3.2+/-1.2$ 
agents among 12 agents or classes for which routine DST was performed, 20) median duration of treatment with injectable agents : 15.4 months, and 21) median duration of treatment ranged 8.0- 24.9 months, median duration from treatment initiation to surgery : 11.6 months, and median duration of treatment for patients undergoing surgery : 31.2 months [70]. The median duration of follow-up was 19.4 months [71]. Treatment outcomes revealed that $60.4 \%$ of patients were cured or completed treatment [71]. This study is currently the up-to-date information of XDR-TB treatment. Positive AFB-smear, and urban residence could be predictors of poor treatment outcomes in XDR-TB [70]. For patients with mono/poly-resistant drug (s) TB, the recommended treatment regimens are shown in the Table 4 (13).

\begin{tabular}{|c|c|}
\hline Mono/Poly-resistant Drug (s) & Regimen \\
\hline Rifampicin mono-resistance & $\begin{array}{l}\text { initial } 2 \text { months of isoniazid, pyrazinamide, and ethambutol, and followed by } \\
\text { 10-16 months of isoniazid, ethambutol and a fluoroquinolone }+/ \text { - initial } 6 \\
\text { months of an injectable drug }\end{array}$ \\
\hline Isoniazid mono-resistance & $\begin{array}{l}\text { initial } 2 \text { months of rifampicin, pyrazinamide, and ethambutol, and followed } \\
\text { by } 4-7 \text { months of rifampicin, and a fluoroquinolone (750-1,000 mg of } \\
\text { levofloxacin or } 400 \text { mg of moxifloxacin substituted for isoniazid in the } \\
\text { standard 6-month short-course regimen) }\end{array}$ \\
\hline $\begin{array}{l}\text { Rifampicin and pyrazinamide }(+/- \\
\text { streptomycin) resistance }\end{array}$ & $\begin{array}{l}\text { at least the initial 2-3 months of isoniazid, ethambutol, a fluoroquinolone, } \\
\text { and an injectable drug (initial } 6 \text { months if extensive disease) for } 18 \text { months of } \\
\text { total treatment duration }\end{array}$ \\
\hline $\begin{array}{l}\text { Rifampicin and ethambutol (+/- } \\
\text { streptomycin) resistance }\end{array}$ & $\begin{array}{c}\text { at least the initial 2-3 months of isoniazid, pyrazinamide, a fluoroquinolone, } \\
\text { and an injectable drug (initial } 6 \text { months if extensive disease) for } 18 \text { months of } \\
\text { total treatment duration }\end{array}$ \\
\hline Isoniazid and pyrazinamide resistance & $\begin{array}{l}\text { 9-12 months of rifampicin, ethambutol,and a fluoroquinolon (longer if } \\
\text { extensive disease) }\end{array}$ \\
\hline Isoniazid and ethambutol resistance & $\begin{array}{l}\text { 9-12 months of rifampicin, pyrazinamide, and a fluoroquinolone (longer if } \\
\text { extensive disease) }\end{array}$ \\
\hline $\begin{array}{l}\text { Isoniazid, pyrazinamide, and ethambutol } \\
\text { (+/- streptomycin) resistance }\end{array}$ & $\begin{array}{l}\text { at least the initial 2-3 months ( } 6 \text { months if extensive disease) of rifampicin, a } \\
\text { fluoroquinolone, an oral second-line drug, an injectable drug for } 18 \text { months } \\
\text { of total treatment duration }\end{array}$ \\
\hline
\end{tabular}

Table 4. Treatment of patients with mono-drug resistant and poly-drug resistant tuberculosis [13]

\section{MDR/XDR-TB treatment-pipeline agents or compounds in clinical trials and related innovative researches}

Currently, drugs in phase III clinical trials are moxifloxacin, gatifloxacin, and meropenem [72]. Heteronemin, nephalsterol, litosterol, and kahalalides are other interesting compounds which are in pre-clinical stage [72]. Okada $\mathrm{M}$ et al. conducted a study on granulysin and a new DNA 
vaccine against MDR/XDR-TB and revealed that agglutinating virus of Japan/Heat-ShockProtein65DNA+Interleukin-12-12DNA vaccine provided strong therapeutic efficacy in killing MDR/XDR-TB bacilli in mice and monkey models [73]. A recent experiment using MDR-TB monkey models which received normal and genetically altered Bacilli Calmette Gue' rin (BCG) vaccines demonstrated that these 2 groups of monkeys survived well compared to the control group [74]. Another study in XDR-TB mice model showed ability of interleukin-7 to kill the bacilli [74].

\section{Totally drug-resistant tuberculosis}

Totally drug-resistant tuberculosis (TDR-TB or XXDR-TB) was recently defined as TB bacilli which resist to all first-line and the 6 second-line drugs (para-aminosalicylic acid, fluoroquinolones, aminoglycosides, thiamines, polypeptides, and cycloserine) [75]. Meanwhile, a recent report from the US-CDC listed 7 challenges that should be addressed before new terminology of TDR-TB should be considered for adopting [76], following are the challenges:

1. The definition should not hinge on resistance to all drugs tested, because the number of drugs tested varies widely between laboratories.

2. In vitro testing data suggest cross-resistance among different drugs within a class of drugs or closely related classes of drugs (e.g., polypeptides and aminoglycosides) is not $100 \%$.

3. Research and reference laboratories in many countries do not test for resistance to the third-line drugs (linezolid, thioridazine, other phenothiazines, monobactams (meropenem, imipenem), macrolides, metronidazole and other imidazoles, clofazimine, and amoxicillin/clavulanic acid).

4. DST for several anti-TB drugs is not sufficiently reliable or reproducible; retesting the same isolate provides a different result in many cases.

5. There are several new anti-TB agents in development pipeline that will be prototypes for new classes of antimycobacterial drugs or add new chemical entities to existing class.

6. Avoiding the unintended implication that patients with TDR-TB should not or cannot be treated.

7. Global laboratory capacity for DST of Mycobacterium tuberculosis isolates remains limited [75]. Two cases of TDR-TB with controversies of terminology and treatment occurred in 2003 in Italy and firstly reported in 2007 [77]. Currently, in Thailand, patients with all antiTB drug-resistance will be prescribed isoniazid alone for lifelong whereas no standardized treatment is recommended yet. During 21-22 March 2012, the WHO had convened a technical consultation to discuss the feasibility and implications of a definition to cover more advanced patterns of TB resistance than XDR-TB [78]. The WHO concluded that reports of severe patterns of anti-TB drug resistance ( worse than XDR-TB alone) are increasing whereas a new definition of anti-TB drug resistance beyond XDR-TB is not recommended [78]. This undefined resistance patterns contributed to technical difficulties 
with DST of several anti-TB agents, the lack of standardized DST methods for several present and new investigational drugs, and insufficient evidence to link such DST results to patients' treatment outcomes [78]. No DST methods for group 5 and new investigational agents currently exist [78]. Molecular DST for the second-line drugs cannot yet replace phenotypic methods [78]. Collaboration between the national TB control programme, Ministries of Public Health, and the pharmaceutical companies will be required to resolve the limitations of treatment options by the compassionate use of new anti-TB agents [78]. The WHO will be the lead in ensuring that better patient data provide a more robust information for future policy decision [78].

\section{Conclusion}

As countries are purchasing and using second-line drugs, the likelihood of misuse and developing of TB-resistant strains increases. Currently, WHO and its partners have reached the phase of expanding MDR-TB control as a component of a comprehensive TB control programme. Launching in 2002, the Global Fund to Fight AIDS, Tuberculosis and Malaria (GFATM) expected that requests for second-line drugs for MDR-TB management should go through the Green Light Committee to prevent their misuse. The number of Green Light Committee-approved MDR-TB control programme is increasing rapidly as a result of main streaming of MDR-TB management into general TB control efforts. Expanding projects and accelerating evidence gathering are essential to further develop international policies. The TBendemic countries themselves and the ability of the technical agencies, as well as the donor community are the factors of future success to expand MDR-TB control programmes.

\section{Author details}

Attapon Cheepsattayakorn*

Address all correspondence to: attaponche@yahoo.com

$10^{\text {th }}$ Zonal Tuberculosis and Chest Disease Centre, Chiang Mai, $10^{\text {th }}$ Office of Disease Prevention and Control, Chiang Mai, Department of Disease Control, Ministry of Public Health, Thailand, Thailand

\section{References}

[1] The WHO/IUATLD Global Project on Anti-tuberculosis Drug Resistance Surveillance. Anti-tuberculosis drug resistance in the world 1999-2002. Third Global Report. Communicable Diseases. Geneva : World Health Organization ; 2003. 
[2] The Bureau of Tuberculosis of Thailand' s Project on Anti-tuberculosis Drug Resistance Surveillance. Anti-tuberculosis drug resistance in Thailand 1997-1998. First Country Report. Nonthaburi, Thailand : Department of Disease Control, Ministry of Public Health; 1999.

[3] The Bureau of Tuberculosis of Thailand 's Project on Anti-tuberculosis Drug Resistance Surveillance. Anti-tuberculosis drug resistance in Thailand 2001-2002. Second Country Report. Nonthaburi, Thailand : Department of Disease Control, Ministry of Public Health; 2003.

[4] The Bureau of Tuberculosis of Thailand 's Project on Anti-tuberculosis Drug Resistance Surveillance. Anti-tuberculosis drug resistance in Thailand 2005-2006. Third Country Report. Nonthaburi, Thailand : Department of Disease Control, Ministry of Public Health; 2007.

[5] Tuberculosis Annual Report-2008. Bangkok, Thailand: The Bureau of Tuberculosis of Thailand ; 2009.

[6] Tuberculosis Annual Report-2003. Bangkok, Thailand: The Bureau of Tuberculosis of Thailand ; 2004.

[7] Tuberculosis Annual Report-2011. Chiang Mai, Thailand : Tenth Zonal Tuberculosis and Chest Disease Centre, Tenth Office of Disease Prevention and Control, Chiang Mai, Thailand; 2012.

[8] Tuberculosis Annual Report-2009. Chiang Mai, Thailand : Tenth Zonal Tuberculosis and Chest Disease Centre, Tenth Office of Disease Prevention and Control, Chiang Mai, Thailand; 2010.

[9] The Bureau of Tuberculosis of Thailand 's Research Project on Anti-tuberculosis Drug Resistance Surveillance. Situation of Multidrug-Resistant Tuberculosis in Thailand : Fiscal Year 2007-2009. Country Report. Nonthaburi, Thailand : Department of Disease Control, Ministry of Public Health; 2010.

[10] Scano F, Vitoria M, Burman W, Harries AD, Gilks CF, Havlir D. Management of HIVinfected patients with MDR- and XDR-TB in resource-limited settings. Int J Tuberc Lung Dis 2008; 12 (12) : 1370-1375.

[11] Jain A, Dixit P. Multidrug-resistant to extensively drug-resistant tuberculosis : what is next? J Biosci 2008 ; 33 (4) : 605-616.

[12] Gillespie SH. Evolution of drug resistance in Mycobacterium tuberculosis : clinical and molecular perspective. Antimicrob Agents Chemother 2002; 46 (2) : 267-274.

[13] Pinto L, Menzies D. Treatment of drug-resistant tuberculosis. Infect Drug Resist 2011; 4: 129-135.

[14] Prasad R. Management of multidrug-resistant tuberculosis : practitioner' s view point. Indian J Tuberc 2007; 54 (1) : 3-11. 
[15] Liu CH, Li HM, Li L, Hu YL, Wang Q, Yang N, et al. Anti-tuberculosis drug resistance patterns and trends in a tuberculosis referral hospital, 1997-2009. Epidemiol Infect 2011; 139 (12) : 1909-1918.

[16] Chakroborty A. Drug-resistant tuberculosis : an insurmountable epidemic ? Inflammopharmacology 2011; 19 (3) : 131-137.

[17] Arnadottir T. The Styblo model 20 years later : what holds true ? Int J Tuberc Lung Dis 2009; 13 (6) : 672-690.

[18] Gandhi NR, Andrews JR, Brust JC, Montreuil R, Weissman D, Heo M, et al. Risk factors for mortality among MDR- and XDR-TB patients in a high HIV prevalence setting. Int J Tuberc Lung Dis 2012; 16 (1) : 90-97.

[19] Marahatta SB. Multidrug-resistant tuberculosis burden and risk factors : an update. Kathmandu Univ Med J (KUMJ) 2010; 8 (29) : 116-125.

[20] Banerjee R, Schecter GF, Flood J, Porco TC. Extensively drug-resistant tuberculosis : new strains, new challenges. Expert Rev Anti Infect Ther 2008; 6 (5) : 713-724.

[21] Prasad R. Multidrug and extensively drug-resistant TB (M/XDR-TB) : problems and solutions. Indian J Tuberc 2010; 57 (4) : 180-191.

[22] Blomberg B. Antimicrobial resistance in developing countries. Tidsskr Nor Laegeforen $2008 ; 128$ (21) : 2462-2466.

[23] Eurosurveillance editorial team. New WHO Europe Action Plan to fight MDR-TB. http//www.eurosurveillance.org/ViewArticle.aspx?Articleld=199967 (accessed 4 August 2012).

[24] The Thailand' s 2012 National Tuberculosis Management Guidelines. Bangkok, Thailand : The Bureau of Tuberculosis of Thailand; 2012.

[25] Singh P, Katoch VM. Multidrug-resistant tuberculosis : current status and emerging tools for its management in India. J Commun Dis 2006; 38 (3) : 216-229.

[26] O' Grady J, Maeurer M, Mwaba P, Kapata N, Bates M, Hoelscher M, et al. New and improved diagnostics for detection of drug-resistant pulmonary tuberculosis. Curr Opin Pulm Med 2011; 17 (3) : 134-141.

[27] Nodieva A, Jansone I, Broka L, Pole I, Skenders G, Baumanis V. Recent nosocomial transmission and genotypes of multidrug-resistant Mycobacterium tuberculosis. Int J Tuberc Lung Dis 2010; 14 (4) : 427-433.

[28] Bodmer T, Strhle A. Diagnosing pulmonary tuberculosis with the Xpert MTB/RIF test. J Vis Exp 2012; 9 (62) : e3547. DOI : 10.3791/3547

[29] Syed J. The tuberculosis diagnostic pipeline. In : Clayden P, Chon L, Collins S, Harrington M, Jefferys R, Jimenez E, Morgan S, Swan T, Syed J, Wingfield C. (eds.) Treatment Action Group. HIV, tuberculosis, and viral hepatitis drugs, diagnosis, vaccines, immune-based therapies, and preventive technologies in development. 
London : TAG; 2010. p107-124. http://www.treatmentactiongroup.org (accessed 31 August 2012).

[30] Mishra B, Rockey SM, Gupta S, Srinivasa H, Muralidharan S. Multidrug-resistant tuberculosis : the experience of an urban tertiary care hospital in South India using automated BACTEC 460 TB. Trop Doct 2012; 42 (1) : 35-37.

[31] Deun A Van, Martin A, Palomino JC. Diagnosis of drug-resistant tuberculosis : reliability and rapidity of detection. Int J Tuberc Lung Dis 2010; 14 (2) : 131-140.

[32] Bemer P, Palicova F, Ru®sch-Gerdes S, Drugeon HB, Pfyffer GE. Multicenter evaluation of fully automated BACTEC mycobacteria growth indicator tube 960 system for susceptibility testing of Mycobacterium tuberculosis. J Clin Microbiol 2002; 40 (1) : 150-154.

[33] Dang TM, Nguyen TN, Wolbers M, Vo SK, Hoang TT, Nguyen HD, et al. Evaluation of microscopic observation drug susceptibility assay for diagnosis of multidrug-resistant tuberculosis in Vietnam. BMC Infect Dis 2012 Mar 1; $12: 49$.

[34] Guillerm M, Usdin M, Arkinstall J., editors. Tuberculosis diagnosis and drug sensitivity testing, an overview of the current diagnostic pipeline. Geneva : Me'decins Sans Frontie 'res : Campaign for Access to Essential Medicines; 2006. http://www.access@geneva.msf.org and http://www.accessmed-msf.org (accessed 11 September 2012).

[35] Bonnet M, Gagnidze L, Varaine F, Ramsay A, Githui W, Guerin PJ. Evaluation of FASTPlaqueTB ${ }^{\mathrm{TM}}$ to diagnose smear-negative tuberculosis in a peripheral clinic in Kenya. Int J Tuberc Lung Dis 2009; 13 (9) : 1112-1118.

[36] Piuri M, Jacobs WR Jr, Hatfull GF. Fluoromycobacteriophages for rapid, specific, and sensitive antibiotic susceptibility testing of Mycobacterium tuberculosis. PLoS ONE 2009; 4(3): e4870. DOI: 10.1371/journal.pone.0004870

[37] Rondo'n L, Piuri M, Jacobs WR Jr, Waard Jde, Hatfull GF, Takiff HE. Evaluation of fluoromycobacteriophages for detecting drug resistance in Mycobacterium tuberculosis. J Clin Microbiol 2011; 49 (5) : 1838-1842.

[38] Musa HR, Ambroggi M, Souto A, A๑ngeby KAK. Drug susceptibility testing of Mycobacterium tuberculosis by a nitrate reductase assay applied directly on microscopypositive sputum samples. J Clin Microbiol 2005; 43 (7) : 3159-3161.

[39] Martin A, Paasch F, Von Groll A, Fissette K, Almeida P, Varaine F, et al. Thin-layer agar for detection of resistance to rifampicin, ofloxacin and kanamycin in Mycobacterium tuberculosis isolates. Int J Tuberc Lung Dis 2009; 13 (10) : 1301-1304.

[40] Minion J, Leung E, Menzies, Pai M. Microscopic-observation drug susceptibility and thin layer agar assays for the detection of drug resistant tuberculosis : a systematic review and meta-analysis. Lancet Infect Dis 2010; 10 (10) : 688-698. 
[41] Martin A, Portaels F, Palomino JC. Colorimetric redox-indicator methods for the rapid detection of multidrug resistance in Mycobacterium tuberculosis : a systematic review and meta-analysis. J Antimicrob Chemother 2007; 59 (2) : 175-183.

[42] Martin A, Paasch F, Docx S, Fissette K, Imperiale B, Ribo'n W, et al. Multicenter laboratory validation of the colorimetric-redox indicator (CRI) assay for the rapid detection of extensively drug-resistant (XDR) Mycobacterium tuberculosis. J Antimicrob Chemother 2011; 66 (4) : 827-833.

[43] Rossau R, Traore H, Beenhouwer Hde, Mijs W, Jannes G, Rijk Pde, et al. Evaluation of the INNO-LiPA Rif.TB assay, a reverse hybridization assay for the simultaneous detection of Mycobacterium tuberculosis complex and its resistance to rifampicin. Antimicrob Agents Chemother 1997; 41 (10) : 2093-2098.

[44] Viveiros M, Leandro C, Rodrigues L, Almeida J, Bettencourt R, Couto I, et al. Direct application of the INNO-LiPA Rif.TB line-probe assay for rapid identification of Mycobacterium tuberculosis complex strains and detection of rifampicin resistance in 360 smear-positive respiratory specimens from an area of high incidence of multidrugresistant tuberculosis. J Clin Microbiol 2005; 43 (9) : 4880-4884.

[45] Abebe G, Paasch F, Apers L, Rigouts L, Colebunders R. Tuberculosis-drug resistance testing by molecular methods : opportunities and challenges in resource-limited settings. J Microbiol Methods 2011; 84 (2) : 155-160.

[46] O'Grady J, Bates M, Chilukutu L, Mzyece J, Cheelo B, Chilufya M, et al. Evaluation of the Xpert MTB/RIF assay at a tertiary care referral hospital in a setting where tuberculosis and HIV infection are highly endemic. Clin Infect Dis 2012 Aug 23. [Epub ahead of print].

[47] Rachow A, Zumla A, Heinrich N, Rojas-Ponce G, Mtafya B, Reither K, et al. Rapid and accurate detection of Mycobacterium tuberculosis in sputum samples by Cepheid Xpert MTB/RIF assay-a clinical validation study. PLoS ONE 2011; 6 (6) : e20458. DOI : 10.1371/ journal.pone.0020458

[48] Shimizu Y, Dobashi K, Yoshikawa Y, Yabe S, Higuchi S, Koike Y, et al. Five-antituberculosis drug-resistance genes detection using array system. J Clin Biochem Nutr 2008; 42 (3) : 228-234.

[49] Wilson ML. Recent advances in the laboratory detection of Mycobacterium tuberculosis complex and drug resistance. Clin Infect Dis 2011; 52 (11) : 1350-1355.

[50] Cha J, Lee HY, Lee KS, Koh WJ, Kwon OJ, Yi CA, et al. Radiological findings of extensively drug-resistant pulmonary tuberculosis in non-AIDS adults : comparisons with findings of multidrug-resistant and drug-sensitive tuberculosis. Korean J Radiol 2009; 10 (3) : 207-216.

[51] Lee ES, Park CM, Goo JM, Yim JJ, Kim HR, Lee IS, et al. Computed tomography features of extensively drug-resistant pulmonary tuberculosis in non-HIV-infected patients. J Comput Assist Tomogr 2010; 34 (4) : 559-563. 
[52] Sneag DB, Schaaf HS, Cotton MF, Zar HJ. Failure of chemoprophylaxis with standard antituberculosis agents in child contacts of multidrug-resistant tuberculosis cases. Pediatr Infect Dis J 2007; 26 (12) : 1142-1146.

[53] Ahuja SD, Ashkin D, Avendano M, Banerjee R, Bauer M, Bayona JN, et al. Multidrugresistant pulmonary tuberculosis treatment regimens and patient outcomes : an individual patient data meta-analysis of 9,153 patients. PLoS Med 2012; 9 (8) : e1001300. DOI : 10.1371/journal.pmed.1001300

[54] Falzon D, Jaramillo E, Schünemenn HJ, Arentz M, Bauer M, Bayona J , et al. WHO guidelines for the programmatic management of drug-resistant tuberculosis. Eur Respir J 2011; 38 (3) : 516-528.

[55] Nathanson E, Weezenbeek L-van, Rich ML, Gupta R, Bayona J, Blöndal K, et al. Multidrug-resistant tuberculosis management in resource-limited settings. Emerg Infect Dis 2006; 12 (9) : 1389-1397.

[56] Cheepsattayakorn A, Cheepsattayakorn R. The outcome of tuberculosis control in special high-risk populations in northern Thailand : an observational study. Journal of Health Systems Research 2009; 3 (4) : 558-566.

[57] Joseph P, Desai VBR, Fredrick JS, Ramachandran R, Raman B, Wares F, et al. Outcome of standardized treatment for patients with MDR-TB from Tamil Nadu, India. Indian J Med Res 2011; 133 (5) : 529-534.

[58] Heller T, Lessells RJ, Wallrauch CG, Bärnighausen T, Cooke GS, Mhlongo L, et al. Community-based treatment for multidrug-resistant tuberculosis in rural KwaZuluNatal, South Africa. Int J Tuberc Lung Dis 2010; 14 (4) : 420-426.

[59] Kurbatova EV, Gammino VM, Bayona J, Becerra MC, Danilovitz M, Falzon D, et al. Predictors of sputum culture conversion among patients treated for multidrugresistant tuberculosis. Int J Tuberc Lung Dis 2012; 16 (10) : 1335-1343.

[60] Dheda K, Shean K, Zumla A, Badri M, Streicher EM, Page-Shipp L, et al. Early treatment outcomes and HIV status of patients with extensively drug-resistant tuberculosis in South Africa : a retrospective cohort study. Lancet 2010; 375 (9728) : 1798-1807.

[61] Well CD. Global impact of multidrug-resistant pulmonary tuberculosis among HIVinfected and other immunocompromised hosts : epidemiology, diagnosis, and strategies for management. Curr Infect Dis Rep 2010; 12 (3) : 192-197.

[62] Shenoi SV, Brooks RP, Barbour R, Altice F, Zelterman D, Moll AP, et al. Survival from XDR-TB is associated with modifiable clinical characteristics in rural South Africa. PLoS ONE 2012;7(3):e31786. DOI : 10.1371/journal.pone.0031786

[63] LoBue P. Extensively drug-resistant tuberculosis. Curr Opin Infect Dis 2009; 22 (2) : 167-173.

[64] Monedero I, Caminero JA. MDR-/XDR-TB management : what it was, current standards and what is ahead. Expert Rev Respir Med 2009; 3 (2) : 133-145. 
[65] Monedero I, Caminero JA. Management of multidrug-resistant tuberculosis : an update. Ther Adv Respir Dis 2010; 4 (2) : 117-127.

[66] Schaaf HS, Marais BJ. Management of multidrug-resistant tuberculosis in children : a survival guide for paediatricians. Paediatr Respir Rev 2011; 12 (1) : 31-38.

[67] Mellado Peña MJ, Baquero-Artigao F, Moreno-Perez D; Grupo de Trabajo de Tuberculosis de la Sociedad Española de Infecologia Pedia'trica. Recommendations of the Spanish Society for Pediatric Infectious Diseases (SEIP) on the management of drugresistant tuberculosis. An Pediatr (Barc) 2009; 71 (5) : 447-458.

[68] Ozkutuk N, Surucuoglu S, Gazi H, Coskun M, Ozkutuk A, Ozbakkaloglu B. Secondline drug susceptibilities of multidrug-resistant Mycobacterium tuberculosis isolates in Aegean region-Turkey. Turk J Med Sci 2008; 38 (3) : 245-250.

[69] Jeon DS, Shin DO, Park SK, Seo JE, Seo HS, Cho YS, et al. Treatment outcome and mortality among patients with multidrug-resistant tuberculosis in tuberculosis hospitals of the public sector. J Korean Med Sci 2011; 26 (1) : 33-41.

[70] Kliiman K, Altraja A. Predictors of poor treatment outcome in multi- and extensively drug-resistant pulmonary TB. Eur Respir J 2009; 33 (5) : 1085-1094.

[71] Mitnick CD, Shin SS, Seung KJ, Rich ML, Atwood SS, Furin JJ, et al. Comprehensive treatment of extensively drug-resistant tuberculosis. N Engl J Med 2008; 359 (6) : 563-574.

[72] Cheepsattayakorn A, Cheepsattayakorn R. Novel compounds and drugs and recent patents in treating multidrug-resistant and extensively drug-resistant tuberculosis. Recent Pat Antiinfect Drug Discov 2012; 7 (2) : 141-156.

[73] Okada M, Kita Y, Nakajima T, Kanamura N, Hashimoto S, Nagasawa T, et al. Novel therapeutic vaccine : granulysin and new DNA vaccine against tuberculosis. Human vaccines 2011; 7 Supplement : 60-67.

[74] Institute of Medicine (US) Forum on Drug Discovery, Development, and Translation; Russian Academy of Medical Science. The New Profile of Drug-Resistant Tuberculosis in Russia : A Global and Local Perspective : Summary of a joint Workshop. Washington (DC) : National Academies Press (US); 2011. 6, Treatment of Drug-Resistant TB. http:// www.ncbi.nlm.nih.gov/book/NBK62451/ (accessed 1 October 2012).

[75] Caminero JA. Treatment of tuberculosis according to the different pattern of resistance. Med Clin (Barc) 2010; 134 (4) : 173-181.

[76] Cegielski P, Nunn P, Kurbatova EV, Weyer K, Dalton TL, Wares DF, et al. Challenges and controversies in defining totally drug-resistant tuberculosis. Emerg Infect Dis. http://dx.doi.org/10.3201/eid1811.120256, http://dx.doi.org/10.3201/eid1811.120256, http://www..cdc.gov/Other/disclaimer.html, and http://www.cdc.gov/eid/article/ 18/11/12-0256_article.htm (accessed 1 October 2012). 
[77] Reichman L. Information about tuberculosis. http://www.tbfacts.org/xdr-tb.html (accessed 1 October 2012).

[78] World Health Organization : Totally Drug-Resistant TB : a WHO consultation on the diagnostic definition and treatment options. http://www.who.int/../tb/.../ Report_Meeting_totallydrugresistant... (accessed 1 October 2012). 